\title{
Identification of the clinical and neuroimaging characteristics in children with neuromyelitis optica spectrum disorders: a case series
}

\author{
Zhan Zhang, Hua Zhou, Xinglou Liu, Lingling Liu, Sainan Shu, Feng Fang^ \\ Department of Paediatrics, Tongji Hospital of Tongii Medical College, Huazhong University of Science and Technology, Wuhan, China \\ Contributions: (I) Conception and design: Z Zhang; (II) Administrative support: Z Zhang, F Fang, X Liu; (III) Provision of study materials or patients: \\ All authors; (IV) Collection and assembly of data: Z Zhang; (V) Data analysis and interpretation: Z Zhang; (VI) Manuscript writing: All authors; (VII) \\ Final approval of manuscript: All authors. \\ Correspondence to: Feng Fang; Xinglou Liu. Department of Paediatrics, Tongji Hospital of Tongji Medical College, Huazhong University of Science \\ and Technology, Wuhan, China. Email: ffang56@163.com; liuxinglou@163.com.
}

Background: Childhood neuromyelitis optica spectrum disorders (NMOSDs) may cause visual impairment
and brain or spinal cord damage, and the effects may be permanent if left untreated. Since the incidence of
NMOSD cases in children is relatively low, the understanding of NMOSD among children is inadequate. Methods: This investigation examined the clinical and neuroimaging characteristics of childhood NMOSD. We retrospectively analyzed the clinical information of 11 NMOSD patients admitted to our centre from 2012 to 2021. The disease status was assessed by the Expanded Disability Status Scale (EDSS) score.

Results: The two major symptoms observed in the study cohort were optic neuritis (ON) (9/11) and encephalopathy (7/11). Antibody tests were performed on 8 children, 2 of whom showed serum aquaporin 4 (AQP4) antibody positivity, and another 2 presented with serum myelin oligodendrocyte glycoprotein (MOG) antibody positivity. All patients showed white matter hyperintensity on magnetic resonance imaging (MRI) scans. Interestingly, a rare radiological sign, enlarged perivascular space (PVS), which is more commonly observed in the elderly or adults, was found in 4 participants with more severe clinical manifestations.

Conclusions: While NMOSD in children is less commonly diagnosed through clinical evaluations, the symptoms of $\mathrm{ON}$ and encephalopathy should raise the possibility of the disease. As the diagnosis of NMOSD in children is relatively difficult, enlarged PVS may represent a promising biomarker for the diagnosis and evaluation of NMOSD.

Keywords: Child; neuromyelitis optica spectrum disorders (NMOSDs); enlarged perivascular spaces (PVS); diagnosis; case series

Submitted Jul 23, 2021. Accepted for publication Sep 07, 2021.

doi: $10.21037 / \mathrm{tp}-21-370$

View this article at: https://dx.doi.org/10.21037/tp-21-370

\section{Introduction}

Neuromyelitis optica spectrum disorder (NMOSD) encompasses a group of immune-mediated demyelinating diseases of the central nervous system that are mainly characterized by monophasic or recurrent optic neuritis
$(\mathrm{ON})$ and acute long-segment myelitis. At present, the exact cause of the disease is still not very clear. Many factors such as infection, immunity, and environment may be related to the onset of NMOSD. The incidence of NMOSD ranges from 0.05 to 4 in every 100,000 individuals. Approximately 3-4\%

\footnotetext{
^ ORCID: 0000-0002-4468-6206.
} 
of NMOSD patients develop the disease in childhood (1), and if not diagnosed in a timely manner, the condition may cause visual impairment and brain or spinal cord damage, which can seriously impact their growth and development, as well as their long-term quality of life (2). Therefore, it is vital to gain further insights into the clinical characteristics and improve its early diagnosis. In particular, some neuroimaging characteristics can help early and rapid diagnosis of NMOSD. Clinical and neuroimaging characteristics of children with NMOSD are different from those of adult patients. Unfortunately, due to the small number of childhood cases, there is a paucity of studies related to childhood NMOSD. This current investigation summarized the general clinical characteristics of childhood NMOSD, as well as the related imaging data. We present the following article in accordance with the AME Case Series reporting checklist (available at https://dx.doi. org/10.21037/tp-21-370).

\section{Methods}

This investigation retrospectively analyzed the clinical data of 11 patients with NMOSD who were admitted to our hospital from March 2012 to August 2021. All study protocols were evaluated and approved by the Clinical Trial Ethics Committee of Huazhong University of Science and Technology, which approved our application for exemption from ethical review considering that this is a retrospective clinical study. Patients were included in this study if they met the diagnostic criteria of NMOSD as specified by the international expert group in 2015 (3). With the improvement of these criteria in recent years, the number of children with a clinical diagnosis of NMOSD has increased (4). Informed consent was obtained from the patients' parents or legal guardians. The clinical features including clinical manifestations, laboratory test results, and imaging data were examined at various stages of the disease.

Antibodies against aquaporin-4 (AQP4-IgG) are a highly sensitive and specific serum biomarker for NMOSD (5). Serum and cerebrospinal fluid (CSF) samples were obtained from patients and sent to the Kangshengda Medical Laboratory Co., Ltd. (Kit: AQP4, MOG autoantibody detection kit. Item No.: MT216-16. Reagent manufacturer: Shanxi Maiyuan Bio) to test for the presence of AQP4IgG and myelin oligodendrocyte glycoprotein antibody (MOG-IgG). Patients were then divided into serum AQP4-IgG positive status, AQP4-IgG negative status, or unknown status. The disease status of patients was evaluated using the Expanded Disability Status Scale (EDSS). The evaluation included visual function, brain stem function, vertebral function, cerebellar function, sensory function, bladder rectal function, and brain function such as walking ability and daily life activities. Each system was graded from normal ( 0 point) to exemplifying the most severe defects (5-6 points), and the scores of each system were combined to obtain the comprehensive EDSS score. All MRI scans were performed as part of the initial clinical routine in our institution. In all cerebral MRI (cMRI) scans, a field strength of $3 \mathrm{~T}$ was used. In almost all patients, the following standard sequences were used: $\mathrm{T} 2$-weighted fast spin echo, T1 spin echo, and fluid attenuated inversion recovery (FLAIR).

\section{Statistical analysis}

Binary categorical variables were summarised as counts (percentages). The association between serum complement and EDSS scores in acute phase was tested via the Spearman's rank-order correlation. All 2-tailed probability values $<0.05$ were considered statistically significant. All statistical analyses were performed with SPSS 22.0. The study was conducted in accordance with the Declaration of Helsinki (as revised in 2013).

\section{Results}

\section{Patient information and clinical manifestation}

A total of 11 children were included in the study, all of whom were diagnosed with NMOSD for the first time. Among these children, there were 7 females and 4 males. The median onset age was 85 months. None of the patients presented with a family history of similar diseases, and no vaccination-related incentives were found.

Most (9/11) children presented with ON, mainly manifested as unilateral or bilateral blurred vision and decreased vision. One patient presented with symptoms of eye pain and the latency of visual evoked potential was prolonged, but there was no obvious abnormality on ophthalmologic examination at the initial onset. However, at the first recurrence 22 months after initial onset, the visual acuity of the left eye decreased significantly. Transient blindness was observed in 3 children and two patients presented with ipsilateral eye pain. A total of 7 patients had encephalopathy, which mainly manifested as dizziness, experiencing darkness, unstable walking, syncope, and limb 
Table 1 Baseline characteristics for all 11 children

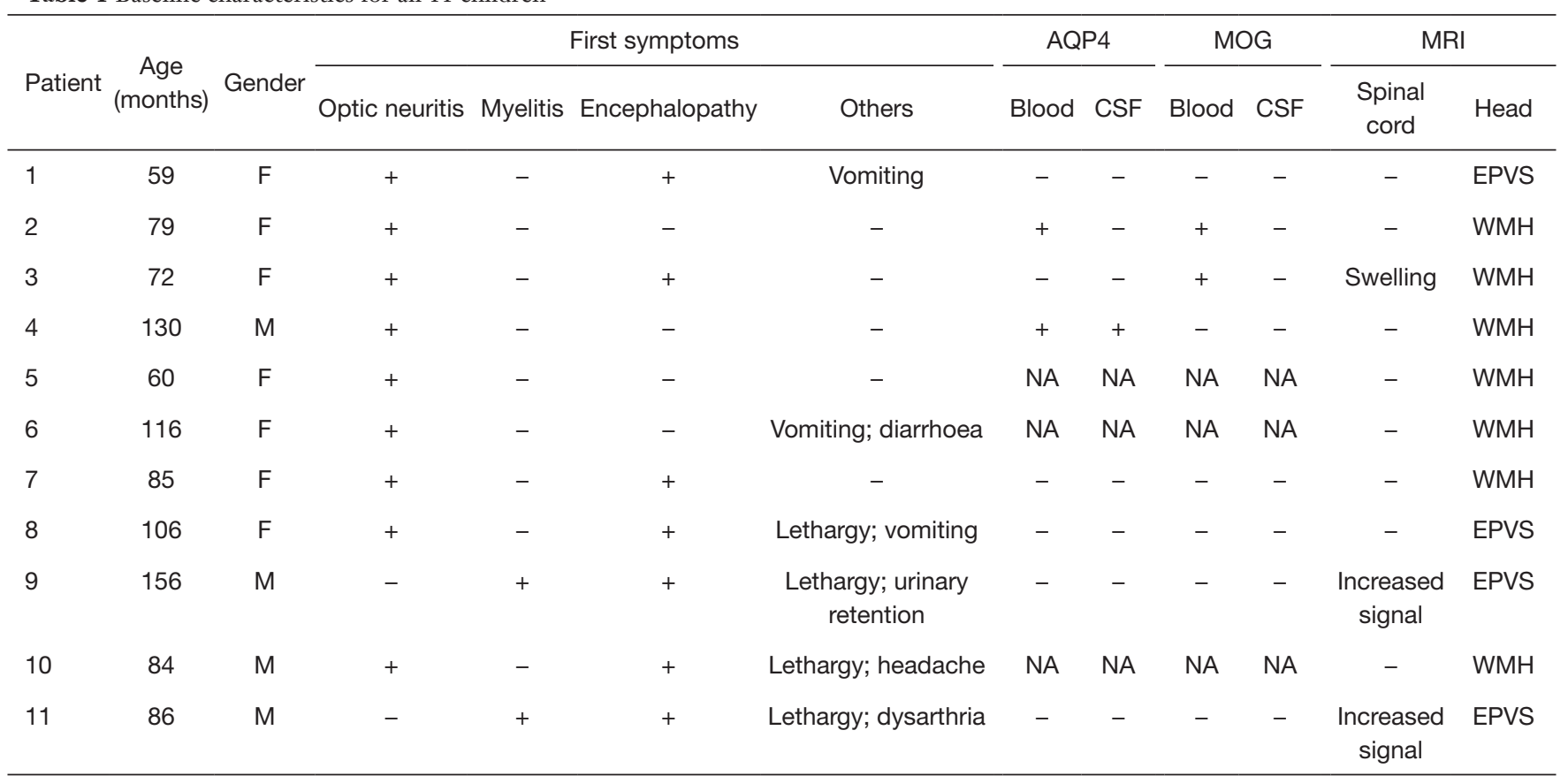

AQP4, aquaporin 4; MOG, myelin oligodendrocyte glycoprotein; MRI, magnetic resonance imaging; CSF, cerebrospinal fluid; M, male; F, female; WMH, white matter hyperintensity; EPVS, enlarged perivascular space; NA, not applicable.

Table 2 Serum complement C4 levels and EDSS scores

\begin{tabular}{lcccccccccc}
\hline Case number & 1 & 2 & 3 & 4 & 5 & 7 & 8 & 9 & 10 \\
\hline Serum C4 (g/L) & 0.27 & 0.35 & 0.11 & 0.12 & NA & 0.14 & 0.12 & 0.25 & 0.29 & 0.21 \\
First EDSS score & 3.5 & 1 & 2 & 4 & 4.5 & 2 & 2 & 1.5 & 1.5 & 1.5 \\
\hline
\end{tabular}

The normal reference range for serum complement in children is 0.16 to $0.38 \mathrm{~g} / \mathrm{L}$. EDSS, Expanded Disability Status Scale; NA, not applicable.

convulsions. There were 4 patients whose cMRI results showed enlarged perivascular spaces (PVS). These children experienced more severe headaches and dizziness, and two showed obvious walking instability. No obvious myelitis manifestations were detected in any of the 8 patients. Nonspecific manifestations included significant vomiting in 3 cases, poor appetite in 2 cases, lethargy in 1 case, dysarthria in 1 case, and diarrhoea in 1 case (Table 1). A total of 10 children underwent EDSS evaluation at the time of first admission and the results are shown in Table 2.

\section{Supplementary examination}

\section{Blood and cerebral spinal fluid testing}

Immune function was examined in 9 patients and there were 4 cases of serum complement $\mathrm{C} 4$ levels that were lower than normal (Table 2). The correlation between serum complement C4 levels and acute EDSS score in children was not statistically significant.

AQP4 and MOG tests were conducted in 8 patients. There were 2 patients who were positive for serum AQP-4 antibodies and 2 patients who were positive for MOG antibodies. One patient's CSF was positive for AQP-4 antibodies (Table 1).

\section{Imaging examination}

All children underwent cMRI scans and all imaging results were abnormal. The main manifestations were different signal lengths, slightly longer T2 signals with different distribution shapes, and slightly higher signals on FLAIR. There were 10 cases with lesions involving the frontal and parietal lobes, 7 cases involving unilateral or bilateral 


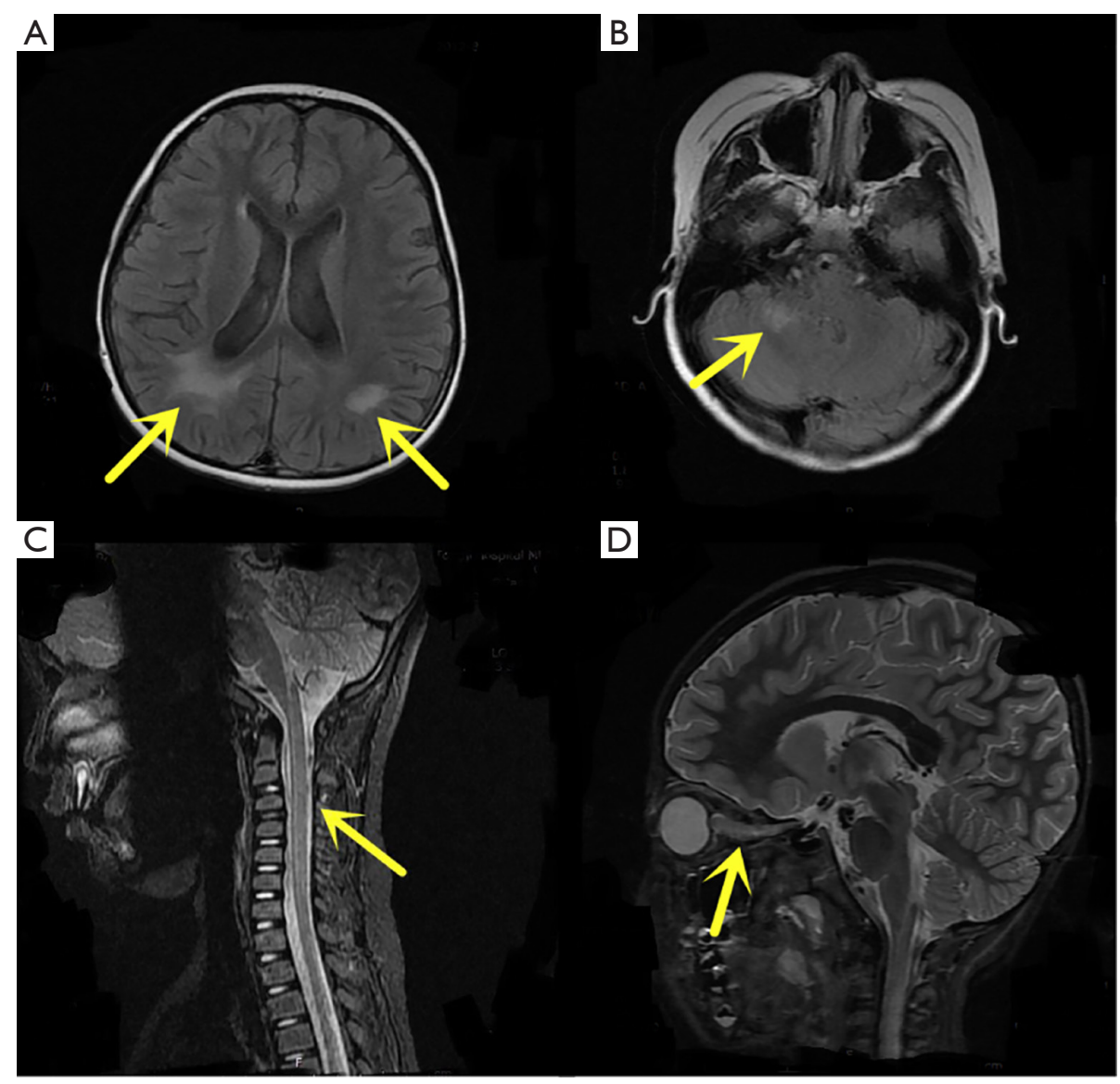

Figure 1 Magnetic resonance imaging of children with neuromyelitis optica spectrum disorders. (A,B) Brain lesions in the white matter and cerebellum of the patients (indicated by the yellow arrows); (C) a spinal MRI of a patient showing a slight swelling of the cervical spinal cord (indicated by the yellow arrow); (D) MRI showing bilateral optic nerve thickening, involving the full length of the optic nerve and the optic chiasm (indicated be the yellow arrow). MRI, magnetic resonance imaging; NMOSD, neuromyelitis optica spectrum disorders.

semioval centres, and 5 cases involving occipital lobes. Spinal MRI was performed in 7 patients, and only 3 had abnormal findings (Figure 1). Enlarged PVS was observed on cMRI in 4 patients, all were localized in the centre of the semioval region, which showed high signal oval or linear lesions on the $\mathrm{T} 2$-weighted image, with a diameter between $1-3 \mathrm{~mm}$ (Figure 2).

\section{Discussion}

In this study cohort, 7 of the 11 participants were female (63.6\%), which is close to the gender distribution reported in previous studies (6). Although the incidence of NMOSD is quite different in men and women, a large-scale study showed no significant difference between males and females in disease onset form, long-term EDSS score, nor disease outcome (7). None of the patients in this study presented with positive family history, but some cases were reported to have familial clusters (8). Some investigations have reported that $3 \%$ of NMOSD cases showed a pattern of familial cluster (9), suggesting that the disease may have a genetic component. NMOSD can be differentiated from other central nervous system inflammatory diseases including multiple sclerosis and acute disseminated encephalomyelitis through clinical and neuroimaging characteristics, laboratory tests, and other tests. The most common symptom of NMOSD in children is ON, which manifests as decreased visual acuity involving unilateral or bilateral visual field defects. Most children in this study presented with ON. In patient 2, the symptoms of optic nerve inflammation were not obvious at the initial onset, but visual acuity decreased with disease recurrence. This 


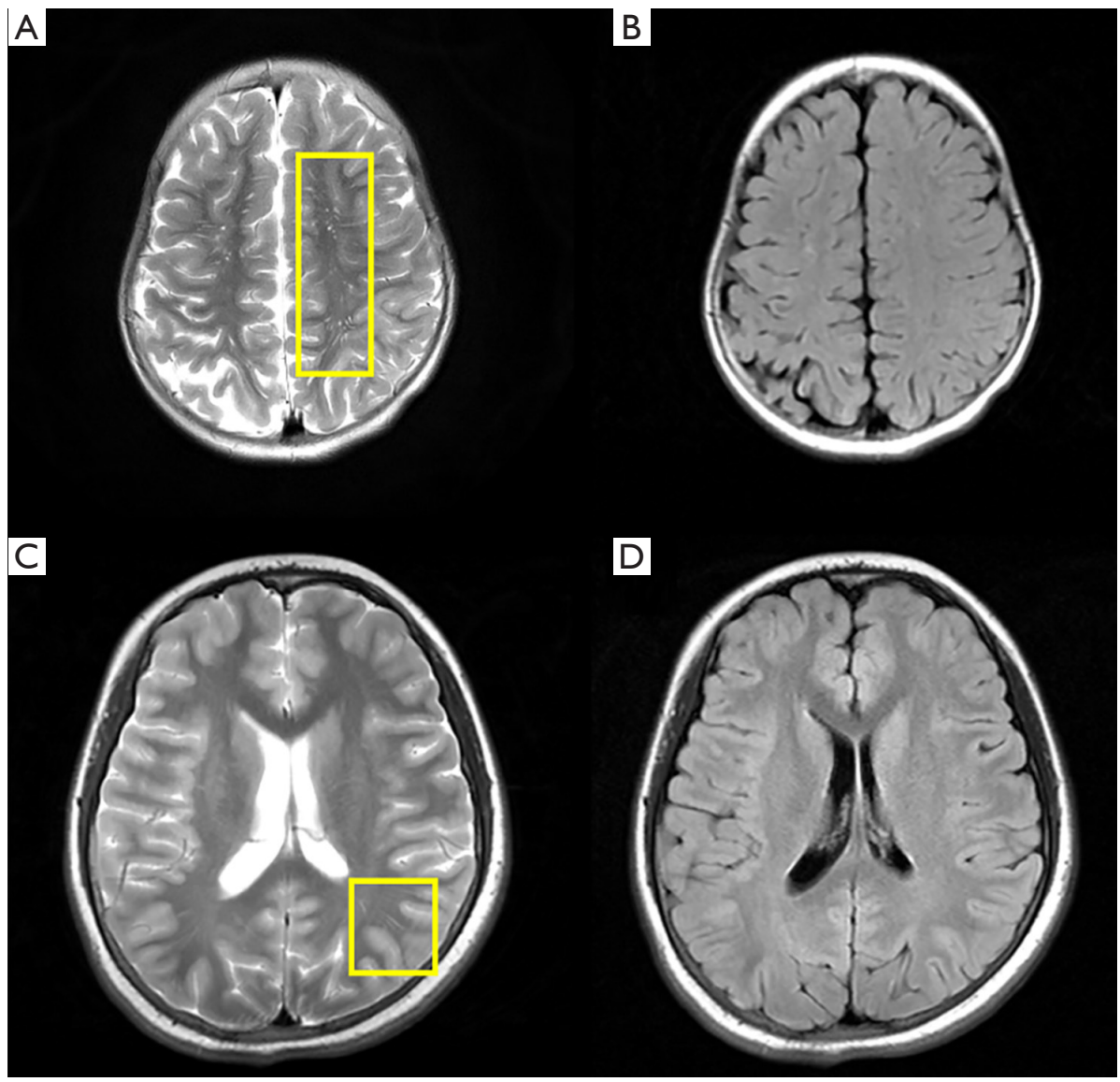

Figure 2 Signs (in the yellow frame) of enlarged perivascular space in children with neuromyelitis optica spectrum disorders. (A,C) T2 scans show enlarged PVS, especially in the centrum semiovale; (B,D) T2-FLAIR scans show hypointense signals in same regions. Note: Images $(A, B)$ were taken from one child, while images (C,D) were taken from another child. PVS, perivascular spaces; FLAIR, fluid attenuated inversion recovery; NMOSD, neuromyelitis optica spectrum disorders.

indicated that some children with NMOSD have mild optic nerve damage at onset, but obvious visual impairment can develop over the course of the disease. A total of 7 patients (63.6\%) experienced encephalopathy, which was significantly higher than the incidence of encephalopathy in adults with NMOSD (25\%) and was consistent with previous studies (10). The main manifestations were dizziness and darkness. Obvious symptoms of myelitis were observed in 2 cases this group of children. There were 3 $(27.3 \%)$ patients with obvious nausea and vomiting. There have been many reports of intractable hiccup and nausea (IHN) in children with NMOSD. In fact, in some cases, $\mathrm{INH}$ is the only manifestation of the disease with any specificity (11).

Since Lennon et al. (12) reported autoantibodies against AQP4 as a specific biomarker for neuromyelitis optica
(NMO) in 2004, serum and CSF AQP4 antibody assays have played a key role in the diagnosis of NMO and NMOSD. In this study, 2 patients had serum anti-AQP4 seropositivity, and 1 patient showed CSF-positivity. The positive detection rate was lower than that reported in other studies (13) and may be related to the sensitivity, specificity, and disease stage of the patient. A decrease in serum complement C4 was observed during the acute phase in some of the children during the study. Previous serum immunological studies in adult NMOSD patients have shown a decrease in serum complement C4 (14) and recent studies have suggested that complement participation is an important part of NMOSD pathogenesis (15). Excessive inflammation can cause increased complement consumption, and therefore, the decreased serum complement C4 levels may be directly related to the intensity of the inflammatory response and 
thus to the severity of the disease. However, the correlation between serum complement C4 levels and acute EDSS score in was not statistically significant which may due to the small sample size.

MRI findings of the head and spinal cord serve as important reference indicators for NMOSD diagnosis. Children with NMOSD have a wide range of brain lesions that sometimes lack specificity. The lesions are mostly in areas with high expression of AQP4, such as the periventricular area, supratentorial area and underlying white matter, midbrain, and the cerebellum. In children who are positive for MOG antibodies, brain damage is more likely to occur around the ventricles, subcortical lesions, and the cortex and near the third ventricle (16). In our study cohort, there were no significant differences in the location and extent of brain lesions between children whose serum and CSF were MOG-positive or MOG-negative. Spinal MRI was performed in 7 patients, and only 3 had abnormal findings, which was significantly different from observations in adults. Most children in this group had symptoms of $\mathrm{ON}$, but only 2 patients had typical optic neuroimaging changes. Other MRI findings in children with more severe ON manifestations, such as transient or permanent blindness, did not show significant changes in the optic nerve. Thus, the severity of optic neuropathy may not be associated with imaging findings.

Interestingly, enlarged PVS was observed in the cMRI scans of 4 NMOSD children (Figure 2). In these cases, this occurred in the common areas, including the basal ganglia, the midbrain, and especially in the centre of the semioval ganglia. The shape was consistent with previous descriptions (17). The PVS has been suggested as a pathway for lymphatic drainage of the brain, containing macrophages, dendritic cells, lymphocytes, and T cells, which may interact with immune cells (18). Although enlarged PVS has been found in a proportion of healthy adults, numerous studies have reported an association between enlarged PVS and adult neurological diseases, such as multiple sclerosis, dementia, inflammation and small vessel disease, cognitive function, and epileptic seizures (19-22). However, there is little evidence of enlarged PVS in healthy children and adolescents (23). Indeed, enlarged PVS in children may reflect the status of diseases such as moyamoya disease, autism, and PTEN hamartoma tumour syndrome (PHTS) (24-26), all of which may cause damage to the cerebral lymphatic system.

The main pathophysiology of NMOSD is the binding of AQP4-IgG to AQP4 in the central nervous system, which then initiates complement- and antibody-dependent cellular cytotoxicity of target astrocytes (27). The localization of AQP4 in astrocytic foot processes is consistent with the role of astrocytes in the development, function, and integrity of the interface of the PVS (28), and thus, it is plausible that the PVS will be affected during the onset of NMOSD. While cases of enlarged PVS in adult NMOSD patients have been reported (29), to the best of our knowledge, this is the first study to report evidence of enlarged PVS in children with NMOSD, which may be indicative of NMOSD progression. Moreover, 4 children with enlarged PVS in our centre showed more severe disease status, with signs of encephalopathy, obvious dizziness, walking instability, and high EDSS scores. Therefore, enlarged PVS may be a potential neuroimaging biomarker for the diagnosis and prognostic assessment of NMOSD in children. However, due to the extremely low incidence of the disease in children, our sample size is small. Future studies involving a larger childhood population are warranted to verify these observations. Histopathological confirmation should also be conducted to confirm the pathophysiology.

This investigation summarized the basic clinical characteristics and identified a new imaging feature in children with NMOSD. This feature may be a potential biomarker for the diagnosis and prognostic evaluation of NMOSD in children. However, future research involving a larger childhood NMOSD population is required to clarify the relationship between enlarged PVS and NMOSD disease status. Furthermore, the precise mechanisms of NMOSD pathogenesis remain to be fully elucidated.

\section{Conclusions}

Childhood NMOSD is mainly characterized by ON and brain lesions. Enlarged PVS is a promising new candidate in the diagnosis and evaluation of this disease. However, more research is warranted to further clarify the relationship between enlarged PVS and NMOSD in children.

\section{Acknowledgments}

Funding: None.

\section{Footnote}

Reporting Checklist: The authors have completed the AME Case Series reporting checklist. Available at https://dx.doi. 
org/10.21037/tp-21-370

Data Sharing Statement: Available at https://dx.doi. org/10.21037/tp-21-370

Conflicts of Interest: All authors have completed the ICMJE uniform disclosure form (available at https://dx.doi. org/10.21037/tp-21-370). The authors have no conflicts of interest to declare.

Ethical Statement: The authors are accountable for all aspects of the work in ensuring that questions related to the accuracy or integrity of any part of the work are appropriately investigated and resolved. All study protocols were evaluated and approved by the Clinical Trial Ethics Committee of Huazhong University of Science and Technology, which approved our application for exemption from ethical review considering that this is a retrospective clinical study. Informed consent was obtained from the patients' parents or legal guardians prior to inclusion in this study. The study was conducted in accordance with the Declaration of Helsinki (as revised in 2013).

Open Access Statement: This is an Open Access article distributed in accordance with the Creative Commons Attribution-NonCommercial-NoDerivs 4.0 International License (CC BY-NC-ND 4.0), which permits the noncommercial replication and distribution of the article with the strict proviso that no changes or edits are made and the original work is properly cited (including links to both the formal publication through the relevant DOI and the license). See: https://creativecommons.org/licenses/by-nc-nd/4.0/.

\section{References}

1. Chitnis T, Ness J, Krupp L, et al. Clinical features of neuromyelitis optica in children: US Network of Pediatric MS Centers report. Neurology 2016;86:245-52.

2. Fragoso YD. Specificities of Children with Multiple Sclerosis and Neuromyelitis Optica. Cent Nerv Syst Agents Med Chem 2018;18:16-20.

3. Wingerchuk DM, Banwell B, Bennett JL, et al. International consensus diagnostic criteria for neuromyelitis optica spectrum disorders. Neurology 2015;85:177-89.

4. Tenembaum S, Yeh EA; Guthy-Jackson Foundation International Clinical Consortium (GJCF-ICC). Pediatric NMOSD: A Review and Position Statement on Approach to Work-Up and Diagnosis. Front Pediatr 2020;8:339.

5. Rostasy K, Reindl M. Role of autoantibodies in acquired inflammatory demyelinating diseases of the central nervous system in children. Neuropediatrics 2013;44:297-301.

6. Fragoso YD, Ferreira ML, Oliveira EM, et al. Neuromyelitis optica with onset in childhood and adolescence. Pediatr Neurol 2014;50:66-8.

7. Yin J, Long Y, Shan F, et al. Clinical manifestations of neuromyelitis optica in male and female patients. Neurol Res 2015;37:967-73.

8. Gombolay GY, Chitnis T. Pediatric Neuromyelitis Optica Spectrum Disorders. Curr Treat Options Neurol 2018;20:19.

9. Chuquilin M, Mullaguri N, Weinshenker B. Pediatric familial neuromyelitis optica in two sisters with long term follow-up. J Clin Neurosci 2016;29:183-4.

10. Absoud M, Lim MJ, Appleton R, et al. Paediatric neuromyelitis optica: clinical, MRI of the brain and prognostic features. J Neurol Neurosurg Psychiatry 2015;86:470-2.

11. Numata Y, Uematsu M, Suzuki S, et al. Aquaporin-4 autoimmunity in a child without optic neuritis and myelitis. Brain Dev 2015;37:149-52.

12. Lennon VA, Wingerchuk DM, Kryzer TJ, et al. A serum autoantibody marker of neuromyelitis optica: distinction from multiple sclerosis. Lancet 2004;364:2106-12.

13. Tenembaum S, Chitnis T, Nakashima I, et al. Neuromyelitis optica spectrum disorders in children and adolescents. Neurology 2016;87:S59-66.

14. Chen Y, Li R, Wu AM, et al. The complement and immunoglobulin levels in NMO patients. Neurol Sci 2014;35:215-20.

15. Pittock SJ, Berthele A, Fujihara K, et al. Eculizumab in Aquaporin-4-Positive Neuromyelitis Optica Spectrum Disorder. N Engl J Med 2019;381:614-25.

16. Jurynczyk M, Geraldes R, Probert F, et al. Distinct brain imaging characteristics of autoantibody-mediated CNS conditions and multiple sclerosis. Brain 2017;140:617-27.

17. Wardlaw JM, Smith EE, Biessels GJ, et al. Neuroimaging standards for research into small vessel disease and its contribution to ageing and neurodegeneration. Lancet Neurol 2013;12:822-38.

18. Granberg T, Moridi T, Brand JS, et al. Enlarged perivascular spaces in multiple sclerosis on magnetic resonance imaging: a systematic review and meta-analysis. J Neurol 2020;267:3199-212.

19. Smeijer D, Ikram MK, Hilal S. Enlarged Perivascular Spaces and Dementia: A Systematic Review. J Alzheimers 
Dis 2019;72:247-56.

20. Low A, Mak E, Rowe JB, et al. Inflammation and cerebral small vessel disease: A systematic review. Ageing Res Rev 2019;53:100916.

21. Hilal S, Tan CS, Adams HHH, et al. Enlarged perivascular spaces and cognition: A meta-analysis of 5 populationbased studies. Neurology 2018;91:e832-42.

22. Liu C, Habib T, Salimeen M, et al. Quantification of visible Virchow-Robin spaces for detecting the functional status of the glymphatic system in children with newly diagnosed idiopathic generalized epilepsy. Seizure 2020;78:12-7.

23. Piantino J, Boespflug EL, Schwartz DL, et al. Characterization of MR Imaging-Visible Perivascular Spaces in the White Matter of Healthy Adolescents at 3T. AJNR Am J Neuroradiol 2020;41:2139-45.

24. Suzuki H, Mikami T, Kuribara T, et al. Pathophysiological consideration of medullary streaks on FLAIR imaging in pediatric moyamoya disease. J Neurosurg Pediatr 2017;19:560-6.

25. DiStasio MM, Nagakura I, Nadler MJ, et al. T lymphocytes and cytotoxic astrocyte blebs correlate across autism brains. Ann Neurol 2019;86:885-98.

26. Plamper M, Born M, Gohlke B, et al. Cerebral MRI and Clinical Findings in Children with PTEN Hamartoma Tumor Syndrome: Can Cerebral MRI Scan Help to Establish an Earlier Diagnosis of PHTS in Children? Cells 2020;9:1668.

27. Shimizu F, Schaller KL, Owens GP, et al. Glucoseregulated protein 78 autoantibody associates with bloodbrain barrier disruption in neuromyelitis optica. Sci Transl Med 2017;9:eaai9111.

28. Roemer SF, Parisi JE, Lennon VA, et al. Pattern-specific loss of aquaporin-4 immunoreactivity distinguishes neuromyelitis optica from multiple sclerosis. Brain 2007;130:1194-205.

29. Pekcevik Y, Izbudak I. Perivascular Enhancement in a Patient with Neuromyelitis Optica Spectrum Disease during an Optic Neuritis Attack. J Neuroimaging 2015;25:686-7.

(English Language Editor: J. Teoh)
Cite this article as: Zhang Z, Zhou H, Liu X, Liu L, Shu S, Fang F. Identification of the clinical and neuroimaging characteristics in children with neuromyelitis optica spectrum disorders: a case series. Transl Pediatr 2021;10(10):2459-2466. doi: $10.21037 / \mathrm{tp}-21-370$ 